\title{
Alternating prism exposure causes dual adaptation and generalization to a novel displacement
}

\author{
ROBERT B. WELCH \\ NASA-Ames Research Center, Moffett Field, California \\ and \\ BRUCE BRIDGEMAN, SULEKHA ANAND, and KAITLIN E. BROWMAN \\ University of California, Santa Cruz, California
}

\begin{abstract}
In two experiments, we examined the hypothesis that repeatedly adapting and readapting to two mutually conflicting sensory environments fosters the development of a separate adaptation to each situation (dual adaptation) as well as an increased ability to adapt to a novel displace. ment (adaptive generalization). In the preliminary study, subjects alternated between adapting their visuomotor coordination to 30-diopter prismatic displacement and readapting to normal vision. Dual adaptation was observed by the end of 10 alternation cycles. However, an unconfounded test of adaptive generalization was prevented by an unexpected prism-adaptive shift in preexposure baselines for the dual-adapted subjects. In the primary experiment, the subjects adapted and readapted to opposite 15-diopter displacements for a total of 12 cycles. Both dual adaptation and adaptive generalization to a 30-diopter displacement were obtained. These findings may be understood in terms of serial reversal learning and "learning to learn."
\end{abstract}

Human beings are capable of adapting to a wide variety of optical rearrangements, the most common of which is prismatic displacement (see Welch, 1978). In a typical experiment, subjects point at targets during prism exposure and receive visual feedback about their accuracy. Before and after this phase, they are measured on targetpointing accuracy with normal vision and no feedback. The outcome of this paradigm is both consistent and robust. Typically, accurate preexposure pointing is followed on the first exposure trial by a large prism-induced error which is quickly corrected by the visual feedback. When the prisms are removed at the end of the exposure phase, subjects err in the direction opposite the displacement. The fact that this error, the negative aftereffect, occurs even when subjects are aware that their vision has been returned to normal indicates that the prism-corrective response has become automatic. The negative aftereffect undergoes a very gradual and usually incomplete decline during the postexposure phase, despite the absence of visual feedback.

\footnotetext{
The results of the present investigation were presented by the senior author at the 1991 meeting of the Psychonomic Society (Welch, Bridgeman, Anand, \& Browman, 1991). The authors wish to thank Mark Goettshche for his technical support and James Clifford, Malcolm Cohen, and David Tomko for their comments on earlier drafts of this paper. This research was supported by funding to B.B. by the Air Force Office of Scientific Research (Grant AFOSR-90-0095) and to R.B.W. by the National Aeronautics and Space Administration (Project UPN 199-1612-34). Correspondence and reprint requests may be addressed to R.B.W., NASA-Ames Research Center, Mail Stop 239-11, Moffett Field, CA 94035-1000.
}

Most prism adaptation experiments are terminated after the negative aftereffect has been measured and thus no formal attempt is made to readapt subjects to normal vision. One likely reason for the absence of a readaptation phase is the common belief that prism adaptation is quite fragile and will therefore be completely and irrevocably abolished as soon as subjects leave the testing apparatus. An implication of this assumption is that if they were to return for a later testing session, their adaptation would start from the same baseline as before. However, both anecdotal and experimental observations reveal this assumption to be incorrect. Thus, if observers adapt repeatedly to a sensory rearrangement, with intervening readaptation to normal vision, it appears that they begin to develop a separate adaptation to each situation, a phenomenon we refer to as dual adaptation.

An everyday example of dual adaptation is adjusting to new prescription lenses. After repeatedly donning and removing spectacles, wearers commonly report disappearance of the depth distortions, illusory visual motion, and coordination difficulties they had experienced earlier. A similar outcome is revealed by the comparison between practiced and novice deep-sea divers, the former group experiencing relatively little face-mask-induced visual distortion when first entering the water and very rapid readaptation upon emerging (Kinney, Luria, Weitzman, \& Markowitz, 1970; Luria \& Kinney, 1970; Luria, Kinney, \& Weissman, 1967).

The first and only published attempts to produce dual adaptation appear to have been by Flook and McGonigle (1977) and McGonigle and Flook (1978). Using squirrel 
monkeys and humans, they found evidence of dual adaptation to rightward and leftward prismatic displacements. That is, after several alternation cycles, their subjects were making the transition between the two displacements with less disruption of hand-eye coordination than in the earlier cycles. Unfortunately, these authors generally described their results by means of group percentages of correct target-pointing responses, rather than by a detailed specification of adaptation and readaptation functions.

Given the paucity of experimental data, a systematic examination of dual adaptation and the conditions conducive to it would appear to be warranted. It is also of interest to determine how broadly the phenomenon generalizes. Is it limited to the sensory states between which observers have alternated? Or does it entail a more general strategy (not necessarily conscious) for adapting to a range of sensory rearrangements? We refer to such a potential capacity as adaptive generalization. It would be analogous to a learning set, or "learning to learn" (Harlow, 1949), in which subjects who learn a variety of two-stimulus discrimination tasks become more proficient in solving new examples of this type of problem. The only attempt to examine this possibility with respect to prism adaptation was a limited experiment by Lazar and Van Laer (1968), and their results were negative.

The specific objectives of the present investigation were: (1) to identify and confirm the conditions necessary for the development of dual adaptation, and (2) to test for adaptive generalization. In our first experiment, subjects alternately adapted to prismatic displacement and readapted to normal vision. However, because of the relatively small sample size $(n=7)$ and some procedural inconsistencies, this study must be considered only preliminary. In the primary experiment, the subjects alternated between symmetrically opposite displacements. In both studies, dualadaptation training was followed by a test of adaptive generalization to a novel displacement.

\section{PRELIMINARY EXPERIMENT}

\section{Method}

\section{Subjects}

Seven right-handed undergraduate students (4 males and $3 \mathrm{fe}$ males) at the University of California, Santa Cruz participated in the experiment to fulfill a psychology course requirement. All were experimentally naive and either possessed $20 / 20$ vision or wore contact lenses. The average age was 19.5 years.

The testing apparatus (Figure 1), patterned after a device introduced by Uhlarik (1972), was located in a dimly lit testing cubicle. The subjects sat at a table and faced a hemicylindrical vertical screen, the center of which was located $62 \mathrm{~cm}$ from the corneal surfaces of their eyes. A Kodak 35-mm slide projector directed a beam of light onto a motor-driven mirror, which reflected it onto the screen in the form of a circular visual target with a diameter of $.5^{\circ}$. The orientation of the mirror, and thus the target's location on the screen, was determined by a program run by an IBM PS $2 / 70$ computer, which also controlled the timing of the opening and closing of the projector shutter. The target appeared in each of three pseudorandomly varied locations: straight ahead of the nose, and $5^{\circ}$ to the left and right.

The subjects wore goggles containing binocular Risley variable prisms and bit into a dental wax biteboard. Their task was to point

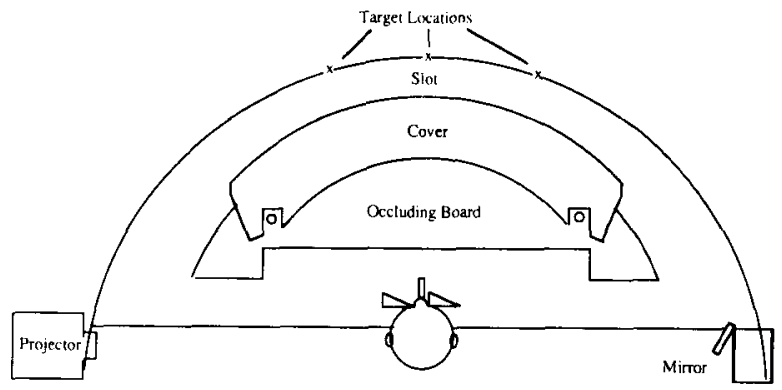

Figure 1. Top view of the testing apparatus, with the slot uncovered.

at the target with a copper stylus which was aligned with the right index finger. From a resting position on the near side of the table, the subjects reached up and forward along the underside of the occluding board until the finger/stylus contacted the screen directly beneath the apparent location of the target. Next, they lowered the finger/stylus until it touched a nichrome resistance wire (not depicted in Figure 1) at the base of the screen. Contact between the stylus and the wire completed an electrical circuit, providing a signal that corresponded to the lateral position of the finger. This position was then recorded and converted by the computer to degrees of visual angle. After each response, the projector shutter was closed, obscuring the target, and the subjects returned the hand to the starting position in preparation for the next response.

A slot located between the far end of the occluding board and the screen was either covered to preclude error-corrective visual feedback or uncovered (as depicted in Figure 1) to provide the subjects with a view of the entire finger/stylus as it touched the screen. The subjects were instructed to point to where the target appeared to be rather than where they knew it was, and to avoid making corrective movements of the finger/stylus while it was visible, even if they missed the target.

\section{Design}

After 18 practice trials with normal vision, Subjects 1-3 (1-day group) completed 10 dual-adaptation cycles $(\mathrm{Cl}-\mathrm{Cl0})$ over a 3 -h period. As seen in Table 1, each cycle consisted of four targetpointing phases: (1) preexposure, which entailed no-displacement, no-feedback measures of target-pointing accuracy; (2) exposure, which entailed exposure to 30 -diopter $\left(17.1^{\circ}\right)$ rightward prismatic displacement with visual feedback and served as a measure of initial prism-induced error and its subsequent attenuation; (3) postexposure, which was identical to the preexposure phase, and measured the negative aftereffect; and (4) readaptation, which entailed normal vision and visual feedback, thereby allowing the subjects to reduce or eliminate the negative aftereffect and thus to readapt to normal vision.

Immediately before each phase, the subjects were informed of the upcoming visual and feedback condition. Between phases, they were to close their eyes and withdraw from the biteboard. A 2to 3-min rest break occurred after Cycles 2, 4, 6, and 8. During this break, the prism goggles were removed and the subjects could leave the testing cubicle and move about freely in an illuminated room or hallway.

Because Subjects 1-3 reported fatigue or extreme tedium in the later stages of the testing period, the procedure was modified for Subjects 4-7 by having them participate in six cycles on 1 day and six cycles 2 days later, with rest periods after Cycles 2, 4, 8, and 10. After the last dual-adaptation cycle on Day 2, these subjects were measured on adaptation to 15-diopter rightward displacement, but were not warned that this displacement would be different from the preceding one. 
Table 1

Phases in a Dual-Adaptation Cycle

\begin{tabular}{lcccc}
\hline & $\begin{array}{c}\text { Preexposure } \\
\text { (Baseline) }\end{array}$ & Exposure & $\begin{array}{c}\text { Postexposure } \\
\text { (Aftereffect) }\end{array}$ & Readaptation \\
\hline Prism & O PD & \pm 30 PD & O PD & O PD \\
Feedback & No & Yes & No & Yes \\
Trials & 18 & 30 & 18 & 30 \\
\hline
\end{tabular}

Note-PD $=$ prism diopter.

\section{Statistical Analyses}

Dual adaptation is evident if the target-pointing error curves for adaptation and readaptation approach baseline more rapidly after a certain number of alternation cycles have occurred than they did early in the training regimen.

The statistical test of dual adaptation involved a phase-by-phase comparison of an early cycle with a late cycle. By means of a repeated measures, cycle $x$ trial analysis of variance (ANOVA) we determined whether the two cycles in a given phase differed reliably with respect to amount (main effect for cycle) and/or pattern of pointing error (the cycle $\times$ trial interaction). Because the statistical effects would be diluted by performance at asymptote, the analysis interval was truncated at the trial on which the subjects had reached asymptote. However, even this analysis is limited because the trial effect is confounded with the error variance. Therefore, the ANOVA was supplemented by a $t$ test in order to examine the difference between corresponding points on the two curves. That is, the datum for a given trial on one curve was subtracted from the datum of the corresponding trial of the other curve and these differences were compared with zero.

\section{Results}

\section{Dual Adaptation}

Because the procedures for the 1- and 2-day groups were virtually identical through Cycle 10 and there was no apparent difference in performance between the groups, they were combined for the statistical analyses. Cycle 10 (the last cycle experienced by all subjects) was compared with Cycle 2 rather than Cycle 1 because preexposure performance on Cycle 2 was found to be biased by a shift in the prism-adaptive direction, apparently the result of the adaptation on the previous cycle. Naturally, such an effect was not obtained on Cycle 1 and thus any comparison of Cycles 1 and 10 with respect to adaptation or readaptation would have been confounded by the difference in preexposure baselines.

The results of this experiment are depicted in Figure 2. It can be seen that the large prism-induced error on the first exposure phase trial for both cycles is followed by a substantial recovery of target-pointing accuracy (Figure $2 b)$. Furthermore, the negative aftereffect curves in the postexposure phase (Figure $2 \mathrm{c}$ ) are approximately flat, whereas the elimination of the negative aftereffect during the readaptation phase (Figure $2 \mathrm{~d}$ ) is both rapid and essentially complete.

Preexposure phase. For this phase (Figure 2a), the cycle $\times$ trial ANOVA failed to produce a significant effect for cycle $[F(1,6)=.04, p>.05]$, trial $[F(17,102)=$

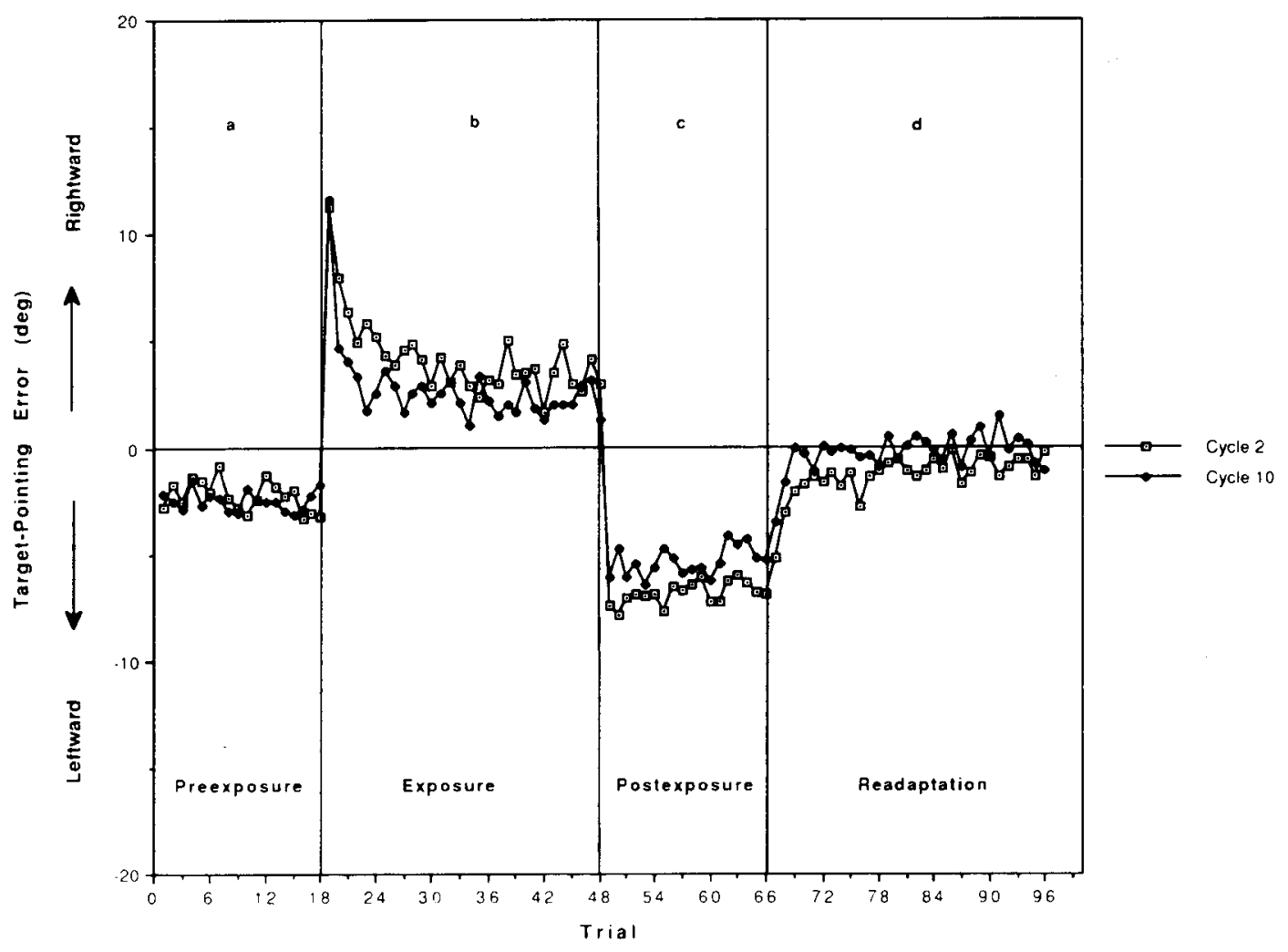

Figure 2. Preliminary study: Target-pointing error (in degrees) for combined 1-day $(n=3)$ and 2-day $(n=4)$ groups on dual-adaptation Cycles 2 and 10 during preexposure (a), exposure (b), postexposure (c), and readaptation (d) phases. 
$1.29, p>.05]$, or their interaction $[F(17,102)=1.21$, $p>.05]$. The failure to find a difference between the cycles in preexposure performance was important because it indicated that later comparisons would be valid. However, as indicated above and seen in Figure 2a, preexposure pointing was not accurate for either of the cycles, but was shifted leftward (the prism-adaptive direction) by $2.26^{\circ}$ for Cycle 2 and $2.5^{\circ}$ for Cycle 10 . Although these shifts were not different from each other, according to correlated-means $t$ tests, they were both different from zero $[t(6)=3.07, p<.03$ for Cycle 2 , and $t(6)=5.40$, $p<.002$ for Cycle 10].

Exposure phase. Visual inspection of the data for this phase (Figure 2b) indicates that the Cycle 10 curve is closer to baseline than is the Cycle 2 curve, suggesting the presence of dual adaptation. For the reasons given in the Methods section, the ANOVA was limited to the first 15 trials. There was neither a main effect of cycle $[F(1,84)=2.43$, $p>.05]$ nor an interaction $[F(14,84)=.41, p>.05]$. However, trial was highly significant $[F(14,84)=87.95$, $p<.001]$. The $t$ test that was applied to all 30 trials produced a significant effect $[t(58)=7.08, p<.001]$.

Postexposure phase. The ANOVA of the postexposure trials (Figure 2c) failed to obtain statistical significance for cycle $[F(1,6)=3.79, p>.05]$, trial $[F(17,102)=$ $1.28, p>.05]$, or their interaction $[F(17,102)=.83$, $p>.05]$. However, once again a $t$ test revealed a statistically significant difference between the two cycles [ $t(34)$ $=14.29, p<.001]$.

Readaptation phase. The ANOVA of the first 15 trials revealed statistically significant effects for trial $[F(14,84)$ $=6.82, p<.001]$ and cycle $[F(1,6)=7.17, p<.05]$, but not for their interaction $[F(14,84)=.94, p>.05]$. Inspection of Figure $2 \mathrm{~d}$ indicates that readaptation was more complete for Cycle 10 than for Cycle 2. As expected, a $t$ test confirmed the effect of cycle $[t(58)=5.51$, $p<.001]$. Although this difference between the curves would appear to offer further support for dual adaptation, it is important to note that it might merely have been a continuation of the difference observed in the preceding phase.

\section{Adaptive Generalization}

To determine whether the training regimen resulted in adaptive generalization, it was necessary to compare the level of adaptation of the trained subjects on the 15-diopter generalization displacement with that of an untrained group. However, the adaptive shift in the preexposure baseline target-pointing performance of the dual-adapted subjects prevented such a comparison. That is, because a control group would not undergo such a shift, it was impossible to determine whether a difference between the two groups on amount or rate of adaptation to the generalization displacement was due to adaptive generalization or merely to a difference in baseline accuracy.

\section{Discussion}

The fact that the Cycle 10 curve was closer to baseline than was the Cycle 2 curve in the exposure, postexposure, and (perhaps) readaptation phases is evidence of dual adaptation. These results encouraged us to implement a second, more systematic, experiment in which we exposed subjects alternately to rightward and leftward prismatic displacement. It was hoped that by using symmetrically opposite displacements, the adaptive shift in preexposure baselines that kept us from making a valid test of adaptive generalization in the preliminary study would be canceled or at least reduced. A second reason for this regimen was to determine whether dual adaptation is limited to situations in which observers alternate between sensory rearrangement and normal perception (as in the preliminary study) or whether it can also occur when both of the environments are rearranged.

\section{PRIMARY EXPERIMENT}

\section{Method}

\section{Subjects}

Six males and 14 females from the same population as in Experiment 1 served as subjects. The average age was 19.3 years.

\section{Design and Procedure}

Ten subjects (experimental group) underwent the dual-adaptation training procedure. Their performance on the generalization test was compared with that of a 10 -subject control group.

After an initial 12-trial target-pointing practice period with normal vision, the subjects underwent two 1.5-h testing sessions which were $48 \mathrm{~h}$ apart. Each session consisted of six cycles of alternation between 15-diopter displacement in one direction and 15-diopter displacement in the opposite direction, with short rest breaks after Cycles 2, 4, 8, and 10 and 2 days' respite between Cycles 6 and 7. Half of the subjects began with rightward displacement and half with leftward displacement.

The phases for a given dual-adaptation cycle are presented in Table 2. The preexposure phase served as a baseline measure of targetpointing accuracy with normal vision and no feedback on the first cycle of each testing session (Cycles 1 and 7) and on the first cycle after each rest break (Cycles 3, 5, 9, and 11). This phase also measured negative aftereffect on the cycles occurring just before the rest breaks or before the end of a testing session (Cycles 2, 4, 8, 10 , and 12). During those times, it was referred to as postexposure

Table 2

Phases for Dual-Adaptation Cycles 1-12

\begin{tabular}{lccccc}
\hline & $\begin{array}{c}\text { Preexposure/ } \\
\text { Postexposure 2 } \\
\text { (Baseline/aftereffect) }\end{array}$ & Exposure & $\begin{array}{c}\text { Postexposure 1 } \\
\text { (Aftereffect) }\end{array}$ & $\begin{array}{c}\text { Reversal } \\
\text { Exposure }\end{array}$ & $\begin{array}{c}\text { Postexposure 2 } \\
\text { (Aftereffect) }\end{array}$ \\
\hline Prism & O PD & \pm 15 PD & 0 PD & \pm 15 PD & 0 PD \\
Feedback & No & Yes & No & Yes & No \\
Trials & 12 & 30 & 12 & 30 & 12 \\
\hline
\end{tabular}

Note-PD $=$ prism diopter. 
Table 3

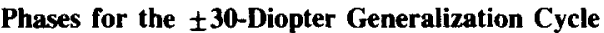

\begin{tabular}{lcccccc}
\hline & $\begin{array}{c}\text { Postexposure 2 } \\
\text { (Aftereffect } \\
\text { from Cycle 12) }\end{array}$ & Readapt 1 & $\begin{array}{c}\text { Preexposure } \\
\text { (Baseline) }\end{array}$ & Exposure & $\begin{array}{c}\text { Postexposure } \\
\text { (Aftereffect) }\end{array}$ & Readapt 2 \\
\hline Prism & O PD & O PD & 0 PD & \pm 30 PD & 0 PD & 0 PD \\
Feedback & No & Yes & No & Yes & No & Yes \\
Trials & 12 & 30 & 12 & 30 & 12 & 30 \\
\hline
\end{tabular}

Note-PD = prism diopter.

Phase 2. During the exposure phase, initial target-pointing error to the 15-diopter prisms and the reduction of this error were assessed. In postexposure Phase 1, the negative aftereffect from the exposure phase was measured. In the reversal exposure phase, the subjects were exposed to prismatic displacement in the direction opposite that of the preceding exposure phase; it was expected that they would make a very large initial error, representing the summation of the negative aftereffect from the previous phase and the prism effect of the current phase. Finally, we come full circle back to postexposure Phase 2 (even-numbered cycles only), in which the negative aftereffect from the reversal phase was measured.

Immediately before each phase, the subjects were informed of whether or not visual feedback would be provided, but were not told of the nature of the sensory condition. Between phases, they were instructed to close their eyes and withdraw from the biteboard.

The experiment concluded on Day 2 with a generalization test cycle (Table 3), which occurred immediately after the 12th (last) dual-adaptation cycle. On this cycle, the negative aftereffect from Cycle 12 was measured in postexposure Phase 2, followed by readaptation to normal vision (readaptation Phase 1 ) and then a preexposure baseline (preexposure phase). This was followed by exposure to 30-diopter displacement (exposure phase) in the direction opposite the last 15-diopter displacement of the dual-adaptation training period. No warning was given to the subjects about the strength of this new displacement. The last 3 subjects of the experimental group were also measured on negative aftereffect for the \pm 30 -diopter displacement (postexposure phase) and the attenuation of this negative aftereffect (readaptation Phase 2).

The control group was exposed to the effects of 30-diopter displacement, with pre- and postexposure measures and a final test of readaptation to normal vision, corresponding to the last four phases of the experimental group (Table 3). For half of these subjects the displacement was to the left, and for the others it was to the right.

\section{Results}

\section{Dual Adaptation}

There were no apparent differences between the experimental subjects whose first prismatic displacement was rightward and those for whom it was leftward, apart from the obvious difference in the direction of prisminduced errors and subsequent adaptation. Therefore, the two sets of data were combined for the analyses by reversing the sign of the target-pointing responses of the leftward-displacement subjects.

As a means of examining the development of dual adaptation over the 12 alternation cycles, we calculated the average of the 30 exposure phase trial scores and the 30 reversal exposure phase scores (with the sign reversed) for each subject and cycle. Figure 3 depicts the group average for this measure for each of the 12 cycles. It is clear from the figure that target-pointing error decreased linearly over the course of the training period.
As in the preliminary study, the statistical test for dual adaptation entailed a comparison between the latest cycle experienced by all the subjects and the first cycle involving an equivalent preceding phase. In the present experiment, the comparison was between Cycles 12 and 2, as depicted in Figure 4. Cycle 2 was used rather than $\mathrm{Cy}-$ cle 1 because the initial baseline measure (i.e., postexposure Phase 2) for the even-numbered cycles represents the negative aftereffect from the preceding phase, and thus only even-numbered cycles can be validly compared. It is evident from the figure that target-pointing accuracy recovered in both cycles during the exposure and reversal exposure phases. Further, as predicted by dual adaptation, the Cycle 12 curve appears to be closer to baseline than is the Cycle 2 curve in the exposure, postexposure, and reversal exposure phases.

Postexposure Phase 2. As indicated above, the shifts away from baseline for the two cycles during this phase (Figure 4a) represent negative aftereffects from the preceding cycle. An ANOVA of these data failed to obtain statistically significant effects for cycle $[F(1,9)=.48$, $p>.05]$, trial $[F(11,99)=1.22, p>.05]$, or their interaction $[F(11,99)=.42, p>.05]$, indicating that the comparison between cycles in the following phase is unconfounded. On the other hand, the slight difference between curves (apparent in Figure 4a) proved statistically significant when analyzed by a $t$ test $[t(22)=5.72, p<.001]$. Fortunately, this difference does not invalidate the comparison between the two cycles in the exposure phase because it is the opposite of the difference between cycles observed in the latter phase. In short, the evidence for dual adaptation in the exposure phase is even stronger when the baseline measures are taken into account.

Exposure phase. An ANOVA on the first 15 trials of this phase (Figure $4 \mathrm{~b}$ ) showed statistical significance for both trial $[F(14,126)=23.26, p<.01]$ and the cycle $\times$ trial interaction $[F(14,126)=2.44, p<.01]$. Although a main effect of cycle was not obtained $[F(1,14)=4.06$, $p>.05]$, a $t$ test indicated that the difference between the two curves (all 30 trials) was statistically significant $[t(58)=6.16, p<.001]$, which is evidence of dual adaptation. The cycle $\times$ trial interaction is indicative of a different pattern of error reduction for the two cycles, with the Cycle 12 curve reaching asymptote in fewer trials than the Cycle 2 curve.

Postexposure Phase 1. According to the ANOVA of the postexposure Phase 1 responses (Figure 4c), trial $[F(11,99)=3.38, p<.001]$ and the cycle $\times$ trial interaction $[F(11,99)=2.58, p<.01]$ were statistically sig- 


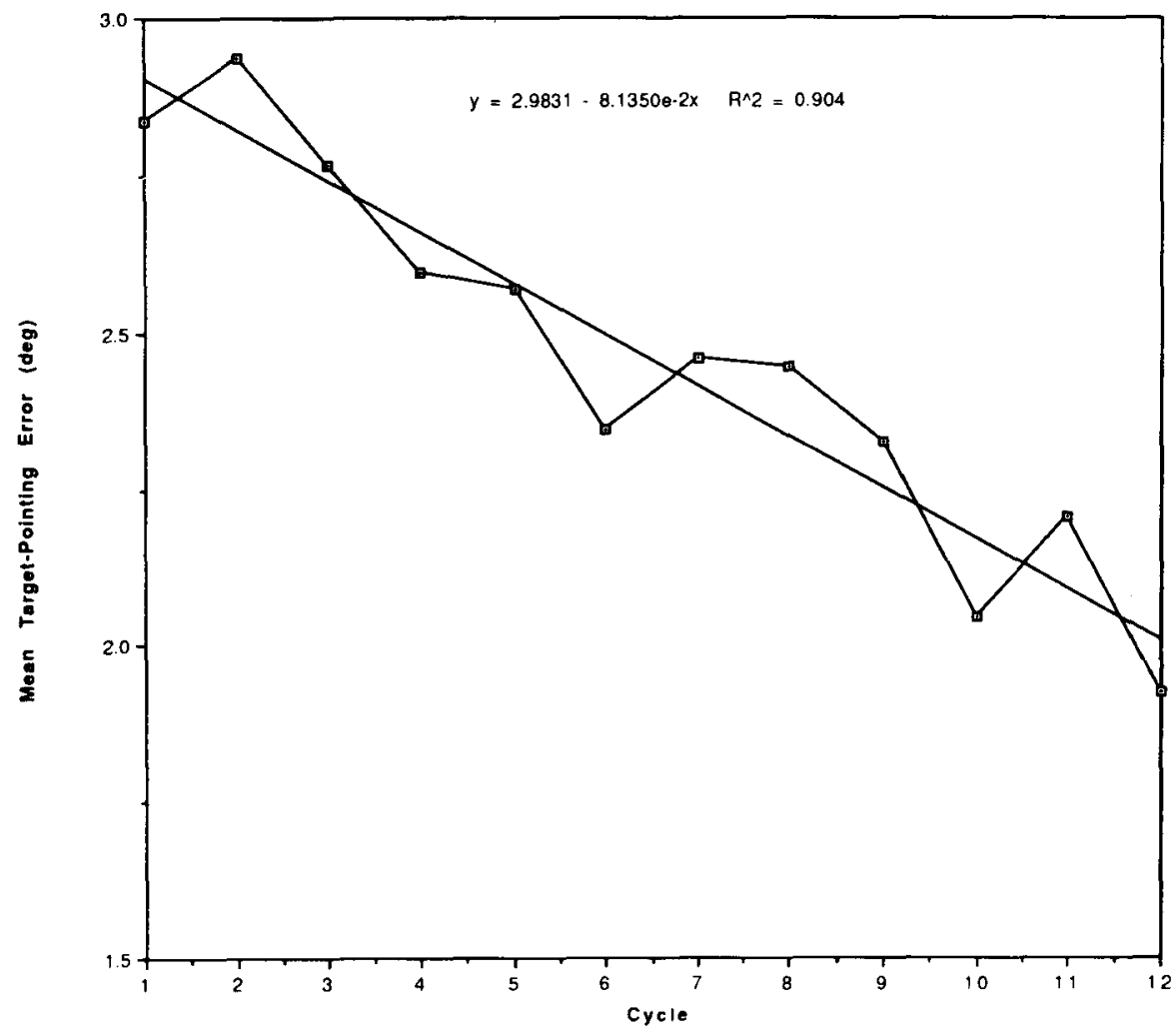

Figure 3. Primary experiment: Average target-pointing error (in degrees) for the experimental subjects on the combined exposure phase trials and the combined reversal exposure phase trials for each dual-adaptation cycle. Note that the sign of the reversal exposure errors was reversed to make them comparable to the exposure phase errors.

nificant, whereas cycle was not $[F(1,11)=2.03, p>$ $.05]$. However, once again, a $t$ test revealed that the difference between the two curves was reliable $[t(22)=9.37$, $p<.001]$. As seen in Figure 4c, target-pointing accuracy on Cycle 12 returned to baseline (despite the absence of visual feedback), whereas the error curve for Cycle 2 remained relatively unchanged.

Reversal exposure phase. It is apparent from Figure 3d that the two curves continue to differ in the final phase in a manner congruent with dual adaptation. According to an ANOVA on the first nine trials, trial $[F(9,72)=$ $8.09, p<.001]$ and cycle $\times$ trial $[F(8,72)=10.60, p<$ $.001]$, were statistically significant. Although there was no main effect for cycle $[F(1,9)=3.86, p>.05]$, the difference did prove statistically significant when examined by means of a $t$ test $[t(58)=6.53, p<.001]$. It should be noted, however, that part or all of the difference between cycles was probably due to the difference in the previous phase. Nevertheless, the cycle $\times$ trial interaction indicates that the Cycle 12 curve approached asymptote more rapidly than the Cycle 2 curve.

\section{Adaptive Generalization}

Figure 5 depicts the target-pointing error for experimental and control groups on the 30-diopter generalization test cycle (see Table 3 ). As predicted by adaptive gen- eralization, the curve for the experimental group is closer to baseline than that for the control group in the exposure, postexposure, and readaptation phases.

Preexposure phase. A group $\times$ trial ANOVA on preexposure phase performance (Figure 4a) revealed a statistically significant effect for group $[F(1,18)=4.67, p<$ $.05]$, but no effect for trial $[F(11,198)=.43, p>.05]$ or for their interaction $[F(11,198)=1.34, p>.05]$. It is important to note that the main effect of cycles for this phase did not invalidate subsequent comparisons because it was in the direction opposite that found in the following phase (see Figures 4a and 4b). Thus, when the exposure phase curves are compared with their respective baselines, support for adaptive generalization is even more compelling.

Exposure phase. An ANOVA on the first 15 trials of this phase (Figure $4 \mathrm{~b}$ ) produced statistically significant effects for group $[F(1,18)=4.69, p<.05]$ and trial $[F(14,252)=6.05, p<.001]$, but not for their interaction $[F(14,252)=.56, p>.05]$. As expected, the difference between groups was confirmed by a $t$ test $[t(58)=$ $75.05, p<.001]$.

Postexposure and readaptation Phase 2. Examination of Figures $4 \mathrm{c}$ and $4 \mathrm{~d}$ suggests that the adaptive generalization of the experimental group in the exposure phase persisted in the form of a much smaller negative after- 


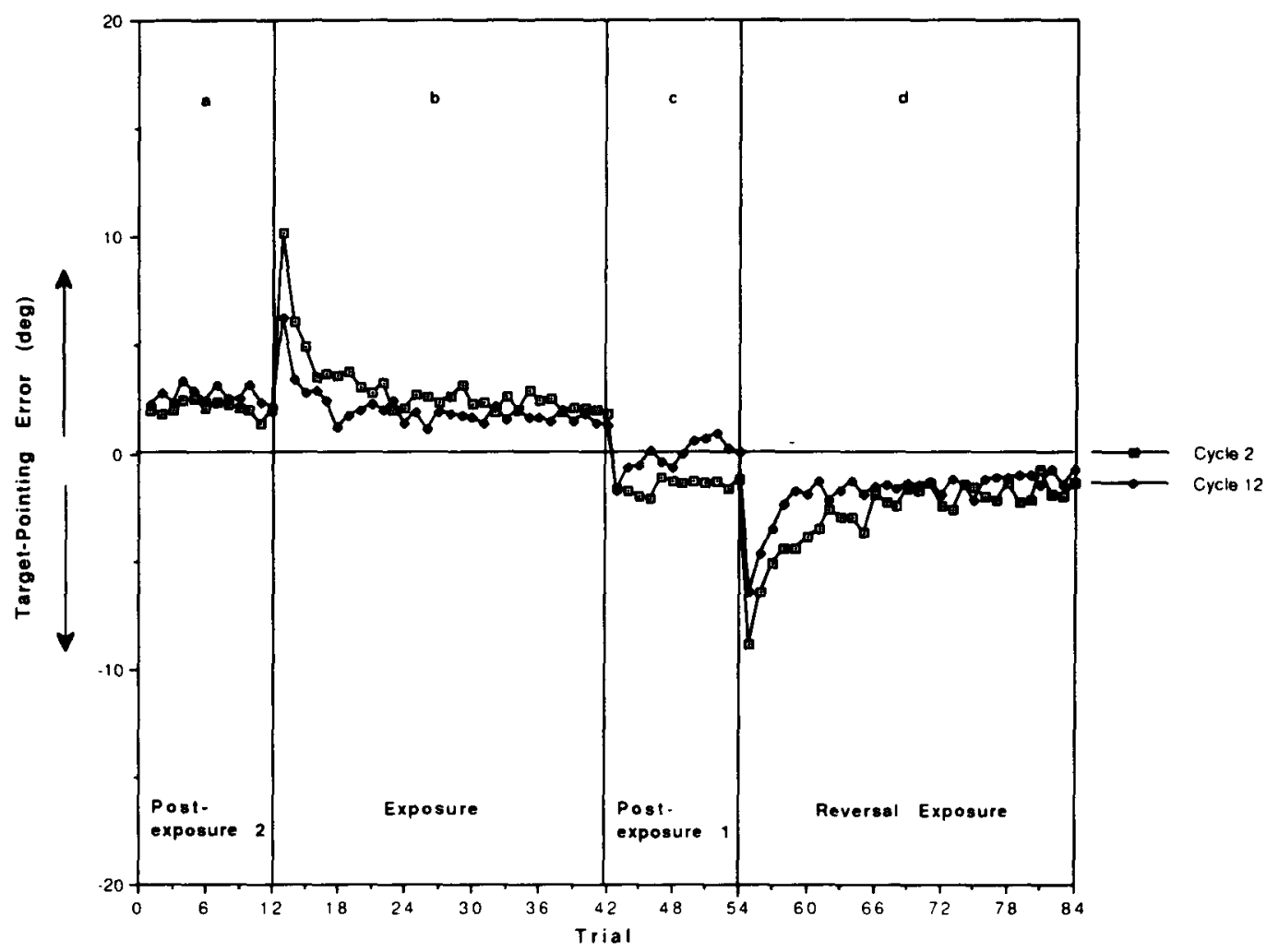

Figure 4. Primary experiment: Target-pointing error (in degrees) for the experimental group on dual-adaptation Cycles 2 and 12 during postexposure Phase 2 (a), exposure phase (b), postexposure Phase 1 (c), and reversal exposure phase (d).

effect (postexposure phase) and reduction of negative aftereffect (readaptation Phase 2). With a sample size of only 3 , however, statistical analysis of these data was inappropriate.

\section{Discussion}

Alternating adaptation and readaptation to \pm 15 -diopter displacement produced dual adaptation in the exposure, postexposure, and readaptation phases of the experiment (Figures $\mathbf{4 b - d}$ ), as well as substantial adaptive generalization to a 30-diopter displacement (Figures 5b-d). Perhaps the most unexpected result of this study was the extreme rapidity with which the negative aftereffect disappeared for Cycle 12 (Figure 4c) and the suggestion of such an outcome for the 3 subjects who were tested on negative aftereffect to the generalization displacement (Figure 5c). It must be assumed that the subjects had learned (perhaps unconsciously) the cues that signaled the onset of normal vision and made the appropriate correction in the postexposure phase, despite the absence of visual feedback. What these cues were is unclear, because no verbal warning was given that vision would be returned to normal for this phase. Perhaps the subjects were able to detect the disappearance of the subtle "side effects" of prismatic displacement (i.e., chromatic aberration, bowing of vertical lines), although none of them reported being conscious of them when questioned in a postexperiment interview. Regardless of the reasons, this result suggests that under some circumstances, the negative aftereffect may greatly underestimate the level of prism adaptation achieved.

\section{GENERAL DISCUSSION AND CONCLUSIONS}

Our data indicate that dual adaptation will appear when subjects alternate (1) between a prismatically displaced and a normal visual field, and (2) between opposite displacements. Furthermore, it was shown in the primary study that a dual-adaptation training regimen can result in adaptive generalization to a novel displacement. Similar findings were independently observed in an unpublished experiment by Bingham, Muchisky, and Romack (1991), using a measure (speed of reaching) quite different from ours. These researchers obtained evidence of dual adaptation, as produced by alternating adaptation to prismatically displaced vision and readaptation to normal vision, as well as enhanced adaptability to a new prismatic displacement.

What is the basis for the dual adaptation and adaptive generalization observed here? One possibility is that repeated adaptation leads to an increased malleability of the relevant sensory system(s). For example, perhaps our dual-adaptation training made it easier to produce an adap- 


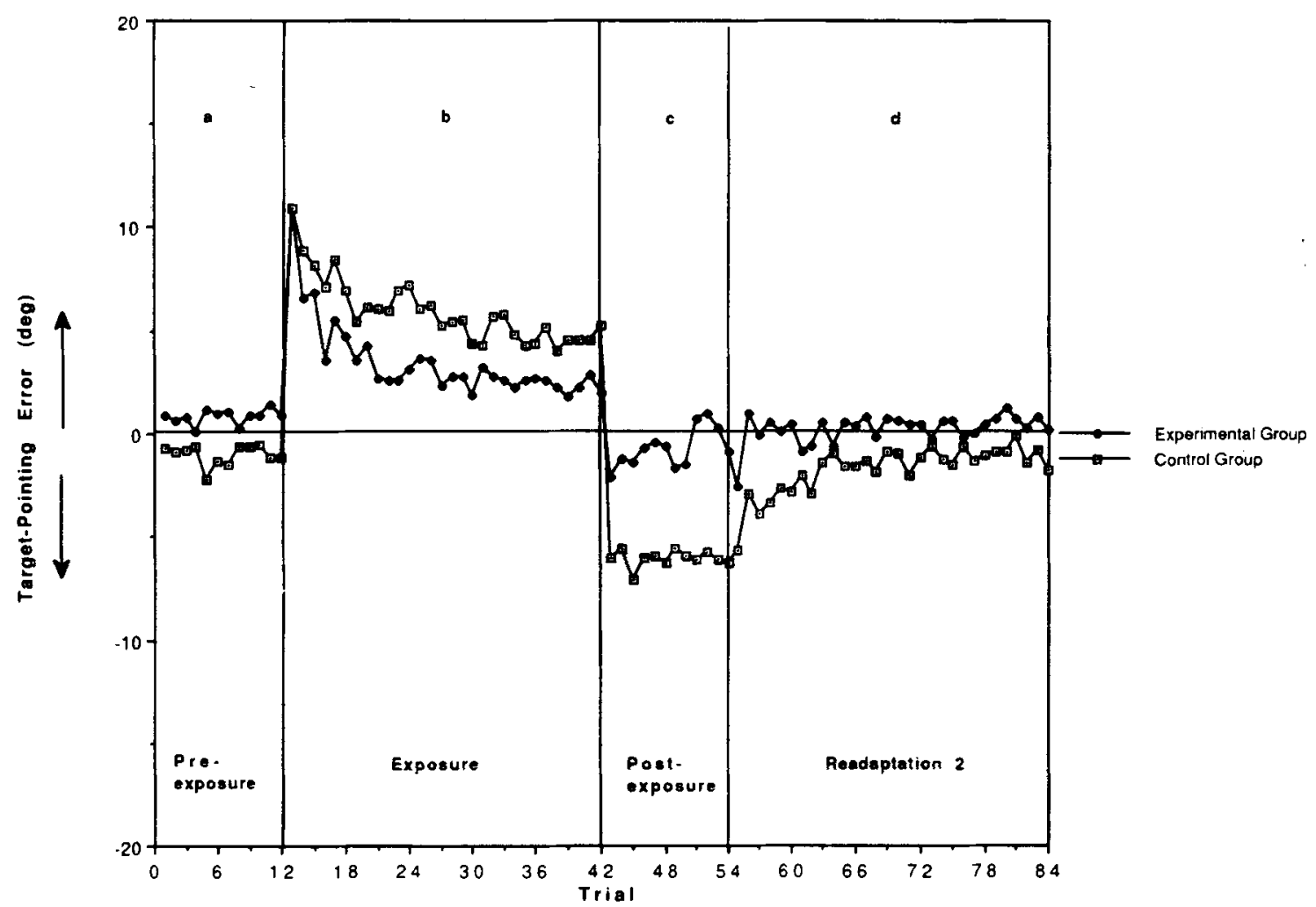

Figure 5. Primary experiment: Target-pointing error (in degrees) for the experimental and control groups on a test of adaptation to 30-diopter prismatic displacement. Note that the data depicted in the postexposure phase and readaptation Phase 2 were obtained from all 10 control subjects, but only 3 experimental subjects.

tive shift in felt limb position (e.g., Harris, 1965). A very different alternative is that the training procedure fostered the development of cognitive strategies for altering handeye coordination. Thus, the subjects may have learned to make rapid shifts in target-pointing strategy as a means of more quickly countering the error produced by the sensory environment that they had just entered or were about to enter. However, if such strategies were used, they were apparently not under conscious control because they were not reported by any of the subjects in the later interview.

There are two ways in which a change in target-pointing strategy might occur. First, cues that reliably predict the transition to the alternate sensory situation might be used by subjects as signals to invoke an adaptive response during the preexposure phase and/or on the very first exposure trial (i.e., before receiving visual feedback). This "premature" adaptive behavior is perhaps best conceptualized as conditioned adaptation (e.g., Kravitz, 1972), in which the predictive stimulus (e.g., removal or replacement of the prism goggles) elicits the adaptive response. Evidence of this form of dual adaptation has been obtained as an incidental and often-ignored result of a variety of studies of adaptation to optical rearrangement, in which subjects were used as their own controls in several conditions, separated by days or weeks (Festinger, Burnham, Ono, \& Bamber, 1967; Gonshor \& Melvill Jones, 1976;
Hein, 1972; Klapp, Nordell, Hoekenga, \& Patton, 1974; Lackner \& Graybiel, 1982; Lackner \& Lobovits, 1977; McLaughlin \& Webster, 1967; Slotnick, 1969; Welch, Choe, \& Heinrich, 1974; Wooster, 1923).

In the second scenario, which is not mutually exclusive to the first, precursor stimuli are unavailable or not used and dual adaptation is manifested only after subjects have first ventured into the alternate sensory world and discovered its characteristics first-hand. Here, the process is analogous to serial reversal learning in which, after a number of reversals of the response-reward contingency on a two-stimulus discrimination task, subjects achieve asymptotic performance immediately after the first trial reveals that the contingency has been reversed (e.g., Harlow, 1949).

Thus, if dual adaptation is based on conditioned adaptation, it should be revealed as an adaptive shift on the baseline measures obtained prior to the exposure phase and/or as a lower intercept of the adaptation and readaptation curves. If dual adaptation is based on serial reversal learning, it should be indicated by steeper adaptation and readaptation curves.

The results of the present investigation provide evidence of both forms of dual adaptation. First, it should be noted that the adaptive shift obtained on the preexposure measures of every cycle after the first in the preliminary study 
(Figure 2a) may be evidence of conditioned adaptation. Also seen in this study were steeper slopes for adaptation and readaptation (Figures $2 b$ and $2 d$ ). In the primary study, dual adaptation took the form of both lower intercepts and steeper curves for adaptation and readaptation (Figures $4 \mathrm{~b}$ and $4 \mathrm{~d}$ ), as well as a steeper curve for the decay of negative aftereffect (Figure $4 c$ ). Finally, the test of adaptive generalization in the primary study revealed a difference in both intercept and slope (Figure 5b).

As already indicated, adaptive generalization is analogous to a general strategy for tackling members of a certain class of problems (i.e., learning to learn). In the present context, this category might be defined as lateral prismatic displacement, although it could be even broader than this. Whether this conceptualization of adaptive generalization is more than analogous remains to be seen. Furthermore, it may be more useful to consider dual adaptation to be merely one manifestation of adaptive generalization, rather than a separate phenomenon.

Dual adaptation and adaptive generalization are evidence that human beings are capable of increasing the rate of their adaptation. However, this raises the question of why they aren't always in this state of advanced adaptability. The answer may be that one pays a price for such a capacity in the form of decreased stability of sensorimotor coordination. One testable prediction from this notion is that dual-adaptation training will cause a subsequent increase in trial-to-trial variability of open-loop target-pointing accuracy. Unfortunately, there were insufficient trials in the present experiment to examine this hypothesis.

The occurrence of dual adaptation has important implications for current attempts to provide astronauts with preflight adaptation training by exposing them, in groundbased simulators, to the visuovestibular and visuomotor discordances they will encounter during weightlessness (e.g., Parker, Reschke, Ouyang, Arrott, \& Lichtenberg, 1986). For practical reasons, such training must be administered weeks or even months before lift-off. However, the existence of dual adaptations suggests that astronauts' capacity for adaptation will remain largely intact during this interval, despite their extensive experience with and readaptation to normal sensory conditions. Furthermore, evidence for adaptive generalization provides a certain amount of confidence that the benefits of preflight training will transfer to microgravity, despite differences in the stimulus characteristics of the two situations.

The present results may also be relevant to aircraft simulator training because they suggest that trainees will be able to acquire separate, nonconflicting adaptations when alternating between a flight simulator and the aircraft that it simulates, between a simulator and the everyday world, and between different simulators. This is important because it implies a reduced problem with "flashbacks," "simulator sickness," and postsimulator mishandling of aircraft and motor vehicles.
Many questions remain about dual adaptation and adaptive generalization, such as how long-lasting they are and by what specific cues observers are able to discriminate the different sensory environments. A question of particular interest concerns whether the dual adaptation obtained in the present investigation is limited to prism-exposure conditions in which error-corrective feedback is provided. Would it occur, for example, when subjects merely move the exposed hand from side to side (e.g., Held \& Gottlieb, 1958)? Such a condition would seem to preclude the adoption of target-pointing strategies, conscious or otherwise, and thereby provide a test of one interpretation of our results.

Another important question concerns how far-ranging adaptive generalization is. For example, in the case of dual-adaptation training involving lateral prismatic displacement, would generalization of adaptation occur to vertical displacement or even right-left reversal of the visual field? We plan to examine these and other related questions.

\section{REFERENCES}

Bingham, G. P., Muchisky, M., Romack, J. L. (1991, November). "Adaptation" to displacement prisms is skill acquisition. Paper presented at the meeting of the Psychonomic Society, San Francisco, CA.

Festinger, L., Burnham, C. A., Ono, H. Bamber, D. (1967). Efference and the conscious experience of perception. Joumal of Experimental Psychology Monograph, 74, (4, Serial No. 637).

Flook, J. P., MCGonigle, B. O. (1977). Serial adaptation to conflicting prismatic rearrangement effects in monkey and man. Perception, 6, 15-29.

Gonshor, A., \& MElvill Jones, G. (1976). Extreme vestibulo-ocular adaptation induced by prolonged optical reversal of vision. Journal of Physiology, 256, 381-414.

Harlow, H. F. (1949). The formation of learning sets. Psychological Review, 56, 51-65.

Harris, C. S. (1965). Perceptual adaptation to inverted, reversed, and displaced vision. Psychological Review, 72, 419-444.

HEIN, A. (1972). Acquiring components of visually guided behavior. In A. D. Pick (Ed.), Minnesota symposia on child psychology (pp. 5368). Minneapolis: University of Minnesota Press.

Held, R., \& Gottlieb, N. (1958). Technique for studying adaptation to disarranged hand-eye coordination. Perceptual \& Motor Skills, 8 , 83-86.

Kinney, J. A. S., Luria, S. M., Weitzman, D. O., \& Markowitz, H. (1970). Effects of diving experience on visual perception under water. (NSMRL Report No. 612). Groton, Connecticut: U.S. Naval Submarine Medical Center.

Klapp, S. T., Nordell, S. A., Hoekenga, K. C., \&atton, C. B. (1974). Long-lasting aftereffects of brief prism exposure. Perception \& Psychophysics, 15, 399-400.

Kravitz, J. H. (1972). Conditioned adaptation to prismatic displacement. Perception \& Psychophysics, 11, 38-42.

LACKNER, J. R., \& GraYbIEL, A. (1982). Rapid perceptual adaptation to high gravitoinertial force levels: Evidence for context-specific adaptation. Aviation, Space, \& Environmental Medicine, 53, 766-769.

LACKNeR, J. R., \& Lobovits, D. (1977). Adaptation to displaced vision: Evidence for prolonged aftereffects. Quarterly Journal of Experimental Psychology, 29, 65-69.

LAZAR, G., \& VAN LAER, J. (1968). Adaptation to displaced vision after experience with lesser displacements. Perceptual \& Motor Skills, 26, $579-582$. 
LuRIA, S. M., \& KInNEy, J. A. S. (1970). Underwater vision. Science, 167, 1454-1461.

Luria, S. M., Kinney, J. A. S., \& Weissman, S. (1967). Estimates of size and distance underwater. American Journal of Psychology, 80, 282-286.

MCGonigle, B. O., Flook, J. P. (1978). Long-term retention of single and multistate prismatic adaptation by humans. Nature, 272 , 364-366.

MCLaughlin, S. C., \& Webster, R. G. (1967). Changes in straightahead eye position during adaptation to wedge prisms. Perception \& Psychophysics, 2, 37-44.

Parker, D. E., Reschke, M. F., Ouyang, L., Arrott, A. P., \& LICHTENBERG, B. K. (1986). Vestibulo-ocular reflex changes following weightlessness and preflight adaptation training. In E. Keller \& D. Zee (Eds.), Adaptive processes in visual and oculomotor systems (pp. 103109). Oxford: Pergamon Press.

SLOTNICK, R. S. (1969). Adaptation to curvature distortion. Journal of Experimental Psychology, 81, 441-448.
UhLARIK, J. J. (1972). A device for presenting targets and recording positioning responses in one dimension. Behavior Research Methods \& Instrumentation, 4, 15-16.

WeLCH, R. B. (1978). Perceptual modification: Adapting to altered sensory environments. New York: Academic Press.

Welch, R. B., Choe, C. S., Heinrich, D. R. (1974). Evidence for a three-component model of prism adaptation. Joumal of Experimental Psychology, 103, 700-705.

Welch, R. B., Bridgeman, B., Anand, S., \& Browman, K. (1991, November). The acquisition of "dual adaptations" and "adaptation sets." Paper presented at the meeting of the Psychonomic Society, San Francisco, CA.

Wooster, M. (1923). Certain factors in the development of a new spatial coordination. Psychological Monographs, 32, (4, Serial No. 146).

(Manuscript received April 6, 1992;

revision accepted for publication January $20,1993$. 